\title{
Constituents of the soft X-ray background
}

\author{
A. M. Sołtan \\ Nicolaus Copernicus Astronomical Center, Bartycka 18, 00-716 Warsaw, Poland \\ e-mail: soltan@camk.edu.pl
}

Received 7 August 2007 / Accepted 16 September 2007

\section{ABSTRACT}

\begin{abstract}
Context. The X-ray background (XRB) is generated by various classes of objects and a variety of emission mechanisms. Relative contribution of individual components depends on energy.

Aims. The goal is to assess the integral emission of the major components of the soft XRB (extragalactic discrete sources dominated by active galactic nuclei (AGN), galactic plasma, and the Warm/Hot Intergalactic Medium [WHIM]), investigating the angular structure of the background.

Methods. Fluctuations of the background are measured using the auto-correlation function of the XRB determined in 5 energy bands between 0.3 and $4.5 \mathrm{keV}$. The investigation is based on the extensive observational data set selected from the XMM-Newton archives. Results. Amplitudes of the auto-correlation functions calculated in three energy bands above $\sim 1 \mathrm{keV}$ are consistent with the conjecture that the background fluctuations result solely from clustering of sources which produce the background. At energies below $1 \mathrm{keV}$ the relative fluctuation amplitude decreases indicating that a fraction of the soft XRB is associated with a smooth plasma emission in the Galaxy. It is shown, however, that the mean spectrum of extragalactic discrete sources steepens in the soft X-rays and is not well represented by a single power law in the energy range $0.3-4.5 \mathrm{keV}$. The WHIM contribution to the total background fluctuations is small and consistent with the WHIM properties derived from the cross-correlation of the XRB with galaxies.
\end{abstract}

Key words. X-rays: diffuse background - intergalactic medium - X-rays: galaxies

\section{Introduction}

The background in the "classic" X-ray domain of 2-8 keV is mostly generated by discrete extragalactic sources (e.g. Lehmann et al. 2001; Kim et al. 2007, and references therein). At lower energies, particularly below $1 \mathrm{keV}$, diffuse hot plasma emission in the Galaxy and the intergalactic space make a detectable contribution (e.g. Henley et al. 2007). The objective of the paper is to assess amplitudes of major X-ray background (XRB) components in different energy bands. The method exploits fluctuation analysis and dependence of the correlation functions of the XRB on the energy band. No assumptions are made on the spectral shape of the individual components.

Time-consuming extensive observations of a large volume of individual objects and precise measurements of the XRB spectrum are considered the straightforward way to determine absolute fluxes of various components contributing to the XRB. However, this method involves detailed modeling of physical parameters of the emitting gas and assumptions on the extragalactic component (cf. McCammon et al. 2002; Henley et al. 2007). In this paper we do not address estimates of the physical status of the galactic and extragalactic media and concentrate on the overall amplitudes of major constituents of the XRB.

The total XRB flux is the sum of several components which have different spatial distributions. Consequently, the resulting XRB fluctuations depend on the relative strength and distribution of each component. In this investigation we distinguish three basic constituents of the XRB. First, the extragalactic point-like sources, which are known to dominate the XRB at higher energies. Fluctuations generated by these objects at angular scales larger than the width of the point spread function (PSF) are described by the relevant auto-correlation function. Second, the galactic emission generated by the Local Bubble and the halo (e.g. Galeazzi et al. 2007). Although the surface brightness distribution of the Local Bubble is highly anisotropic at large angular scale, it is presumed to be smooth at small scales. It is expected that the halo emission is also smoothly distributed. Third, the emission produced by the intergalactic medium.

A major fraction of baryons in the local universe remains in the intergalactic space (e.g. Cen \& Ostriker 1999). In the recent years, physical conditions of this diffuse matter component have been investigated theoretically and examined observationally by several groups. Simulations of interactions between primordial gas falling onto galaxies with galactic wind indicate that some fraction of the diffuse component is heated in shocks and enriched with heavier elements (e.g. Davé et al. 2001; Bryan \& Voigt 2001; Croft et al. 2001). In the areas around concentrations of matter, density of the diffuse component increases substantially and temperatures reach $10^{5}-10^{7} \mathrm{~K}$. Because of characteristic physical parameters, this component of baryonic matter has been termed by Davé et al. (2001) as warm/hot intergalactic medium (WHIM). At temperatures above $\sim 10^{6} \mathrm{~K}$ the WHIM becomes a source of soft X-rays emitted via the thermal Bremsstrahlung mechanism. Thus, the WHIM contributes to the XRB separately from well recognized sources. However, the WHIM signal even at low energies is expected to be small in comparison to the integral XRB surface brightness. It is completely negligible above $2 \mathrm{keV}$. At lower energies the WHIM contribution increases, but using only the spectral characteristics, it is difficult to distinguish this component from the emission by hot plasma in the Galaxy halo.

Spatial distribution of the WHIM is highly nonuniform. Clumpiness of the WHIM emission is a crucial factor which allowed us to isolate and identify this constituent of the XRB and observationally confirm existence of the WHIM itself (Sołtan et al. 2002, 2005). Both the soft spectrum and large linear sizes 
of the emitting regions are consistent with the theoretical predictions for the WHIM clouds based on the hydrodynamical simulations. Observations also confirm theoretical estimates that the WHIM contribution to the integral XRB flux is low even in the soft energy bands (see below). Consequently, the WHIM contribution to the fluctuation amplitude of the integral XRB is also small in comparison to the fluctuations generated by AGNs.

In the present paper the auto-correlation function (ACF) of the total counts, including the non-cosmic contamination is determined in five energy bands covering range $0.3-4.5 \mathrm{keV}$. A relationship between the ACF signal of the raw counts and the intrinsic ACF amplitude of the XRB is obtained. To evaluate a cosmic signal, the ACF requires "suitable" data, corrected for observational and instrumental bias. A question of what sources generate the XRB fluctuations is discussed.

The organization of the paper is as follows. In the next section procedures and criteria applied to the observational material are presented. In Sect. 3 a method to calculate the ACF using a large set of pointing observations is described and the raw results of the calculations are presented. The interpretation of the ACF amplitude distribution over the total investigated energy range is examined in Sect. 4. Basic conclusions of the paper are summarized in Sect. 5.

\section{Observational material}

The X-ray data are extracted from the public archive ${ }^{1}$ of the XMM-Newton EPIC/MOS observations. The pointings are selected in the similar way to that described in the previous papers in this series. For the full description of the data selection and reduction the reader is referred to papers by Sołtan et al. (2005) and Sołtan (2006). Here only the main points are recalled and important modifications of the original procedures described.

All the data obtained with the MOS1 and MOS2 detectors and the thin filter have been inspected, and only pointings without strong sources and known extended sources have been accepted for further processing. Five energy bands as in Sołtan (2006) have been used: $0.3-0.5 \mathrm{keV}, 0.7-1.0 \mathrm{keV}, 1.0-1.35 \mathrm{keV}$, $1.9-3.0 \mathrm{keV}$, and 3.0-4.5 keV. Energy gaps $0.5-0.7 \mathrm{keV}$ and $1.35-1.9 \mathrm{keV}$ reduce confusing Galaxy contribution and strong internal background due to fluorescent lines (Nevalainen et al. 2005). The count distribution for each pointing and the energy band has been corrected for vignetting (Sołtan et al. 2005).

Homogeneity of the data constitutes an obvious criterion which should be satisfied in the ACF calculations. Criteria applied by Sołtan (2006) to select the data for the investigation of the cross-correlation function (CCF) of the XRB and the galaxy distribution are not sufficiently stringent for the ACF calculations. This is because the ACF is highly vulnerable to various instrumental effects which are of lesser importance in the $\mathrm{CCF}$ calculations. In particular, different amounts of the particle background in individual pointings and deficiency in the vignetting corrections have little effect on the CCF amplitude, but generate large spurious ACF signal. The first point is dealt with in the next section. To minimize effects of residual fluctuations due to inaccuracies of the vignetting corrections, we limit the ACF calculations to the central CCD detector in both EPIC MOS cameras. In the central region of the field of view vignetting effects are relatively small, and the procedure which

1 XMM-Newton Science Archive: http://xmm.vilspa.esa.es/external/xmm_data_acc/xsa/ index.shtml corrects the vignetting is more reliable. Moreover, one can expect that the data collected within a single detector are more homogeneous that those from several detectors. Consequently, the data restricted to the central detector are less affected by the instrumental biases and more "suitable" for the fluctuation analysis than all the data recorded in the "Full Frame" mode. Unfortunately, volume of the usable data is much smaller and the results of the present calculations are subject to larger statistical uncertainties.

\section{Auto-correlation function from the scattered pointings}

Constraints imposed on the present observational material severely affect methods of the ACF calculations. First, each pointing observation covers a small area of the sky, roughly $10^{\prime} \times$ $10^{\prime}$. Second, each pointing is contaminated by the unknown a priori number of the non-cosmic counts (mostly charged particles). It implies that the recorded count rates in two observations cannot be used simultaneously to assess the ACF, or - equivalently - the ACF cannot be determined at separations greater than the angular size of the individual pointing.

Let $t_{i}(\boldsymbol{x})$ represents the distribution of count rates on the celestial sphere in the $i$ th observation, where $\boldsymbol{x}$ defines a point on the sphere. The total count rate is a sum of the X-ray counts $\rho_{i}$ and the contaminating background counts $b_{i}$ :

$t_{i}(\boldsymbol{x})=\rho_{i}(\boldsymbol{x})+b_{i}$,

where both the XRB counts and the total counts depend on the position $\boldsymbol{x}$, while the non-X-ray counts $b_{i}$ are randomly distributed in the field of view and are different for each pointing. To estimate the XRB ACF using set of $N$ pointings $(i=1, \ldots, N)$ we define a function $\Delta_{i}(r)$ :

$\Delta_{i}(r) \equiv\left\langle t_{i}(\boldsymbol{x}) \cdot t_{i}(\boldsymbol{x}+\boldsymbol{r})\right\rangle-\left\langle t_{i}\right\rangle^{2}=\left\langle\rho_{i}(\boldsymbol{x}) \cdot \rho_{i}(\boldsymbol{x}+\boldsymbol{r})\right\rangle-\left\langle\rho_{i}\right\rangle^{2}$,

where the angle brackets $\langle\ldots\rangle$ denote the averaging over the field of view of the $i$ th pointing. The right-hand side of the Eq. (2) is now expressed as:

$\Delta_{i}(r)=\frac{1}{A_{i}} \int_{A_{i}} \mathrm{~d}^{2} x \rho_{i}(\boldsymbol{x}) \cdot \rho_{i}(\boldsymbol{x}+\boldsymbol{r})-\left[\frac{1}{A_{i}} \int_{A_{i}} \mathrm{~d}^{2} x \rho_{i}(\boldsymbol{x})\right]^{2}$,

where $A_{i}$ is the area of the $i$ th pointing. We now calculate the average of the relevant quantities in Eqs. (2) and (3) over all the pointings. Equation (3) gives:

$$
\begin{aligned}
\Delta(r) \equiv \frac{1}{N} \sum_{i} \Delta_{i}(r)= & \frac{1}{A} \int_{A} \mathrm{~d}^{2} x\left\langle\rho_{i}(\boldsymbol{x}) \cdot \rho_{i}(\boldsymbol{x}+\boldsymbol{r})\right\rangle \\
& -\frac{1}{A^{2}} \iint_{A} \mathrm{~d}^{2} x_{1} \mathrm{~d}^{2} x_{2}\left\langle\rho_{i}\left(\boldsymbol{x}_{\mathbf{1}}\right) \cdot \rho_{i}\left(\boldsymbol{x}_{\mathbf{2}}\right)\right\rangle .
\end{aligned}
$$

Here the angle brackets $\langle\ldots\rangle$ denote the expectation of the relevant distributions. The average over $N$ pointings on the righthand side of Eq. (4) has been replaced by the overall expectation values integrated over the field of view of a single pointing (all the pointings are of the same shape). Equation (4) can be rewritten in the form:

$\Delta(r)=\langle\rho\rangle^{2}[\xi(r)-I(A)]$, 
where $\langle\rho\rangle$ is the average count rate of the $\mathrm{XRB}, \xi(r)$ is the ACF defined as:

$\xi(r)=\frac{\langle\rho(\boldsymbol{x}) \cdot \rho(\boldsymbol{x}+\boldsymbol{r})\rangle}{\langle\rho\rangle^{2}}-1$,

and $I(A)$ is the average of the $\xi(r)$ over the pointing field of view $A$ :

$I(A)=\frac{1}{A^{2}} \int_{A} \mathrm{~d}^{2} x_{1} \int_{A} \mathrm{~d}^{2} x_{2} \xi\left(r=\left|x_{1}-x_{2}\right|\right)$.

The left-hand side of Eq. (2) averaged over all the pointings is used to define the raw correlation function $w(r)$ :

$w(r)=\frac{\frac{1}{N} \sum_{i}\left\langle t_{i}(\boldsymbol{x}) \cdot t_{i}(\boldsymbol{x}+\boldsymbol{r})\right\rangle-\left\langle t_{i}\right\rangle^{2}}{\langle t\rangle^{2}}$,

where $\left\langle t_{i}\right\rangle$ is the average total count rate in the $i$ th pointing, and $\langle t\rangle$ is the average count rate over all the pointings. Equations (2) and (5) give:

$\xi(r)=\frac{\langle t\rangle^{2}}{\langle\rho\rangle^{2}} w(r)+I(A)$.

Thus, the ACF is undetermined to an arbitrary additive constant $I(A)$. This is because variations of count rates only within a single pointing should be attributed to the actual XRB fluctuations, while differences of the count rates between pointings are partially generated by the non-cosmic signal. To eliminate the unknown parameter $I(A)$, incremental form of Eq. (9) will be used:

$\xi\left(r_{1}\right)-\xi\left(r_{2}\right)=\frac{\langle t\rangle^{2}}{\langle\rho\rangle^{2}}\left[w\left(r_{1}\right)-w\left(r_{2}\right)\right]$,

where both $r_{1}$ and $r_{2}$ are within the field $A$. The $w(r)$ function is presented in the next subsection and implications for the XRB components imposed by the ACF calculated using Eq. (10) are discussed in Sect. 4.

\subsection{The raw correlation function}

Nearly 500 pointings were examined for the present investigation. About 200 pointings, most of them with two MOS cameras, were qualified for further processing. All the point-like sources in the accepted pointings have been removed. The observations were selected at high galactic latitudes, typically above $|b|>30 \mathrm{deg}$, and only the pointings with relatively low hydrogen column density, $N_{\mathrm{H}}$, were used. Threshold values for the $N_{\mathrm{H}}$ adopted in the selection process are given in Table 1. Effects related to the low energy absorption in the Galaxy, albeit small, have been accounted for in the subsequent analysis. The total exposure time of the data used in the calculations exceeded $8 \mathrm{Ms}$. The distributions of counts were binned into $4^{\prime \prime} \times 4^{\prime \prime}$ pixels and the $w(r)$ functions were determined according to Eq. (8). Figure 1 shows results of the computations for separations $30^{\prime \prime}<$ $r<7.5^{\prime}$ and 5 energy bands. The $w(r)$ functions based on MOS 1 and 2 detectors are marked with different symbols. Scatter of points indicates statistical uncertainties due to the limited number of counts, and both detectors give similar results. Possible small deviations of the $w(r)$ distributions between MOS 1 and 2 provide some insight into systematic uncertainties involved in the calculations. All the $w(r)$ distributions exhibit a sharp peak at separations below $\sim 30^{\prime \prime}$ (not shown in plots), generated by the unresolved sources weaker than the detection threshold. Due
Table 1. Raw correlation function increments.

\begin{tabular}{clcc}
\hline \hline$\#$ & $E[\mathrm{keV}]$ & $N_{\mathrm{H} \max }\left[10^{21} \mathrm{~cm}^{-2}\right]$ & $\Delta w$ \\
\hline 1 & $0.3-0.5$ & 0.5 & $(5.66 \pm 0.91) \times 10^{-3}$ \\
2 & $0.7-1.0$ & 1.0 & $(1.16 \pm 0.15) \times 10^{-2}$ \\
3 & $1.0-1.35$ & 2.0 & $(1.34 \pm 0.14) \times 10^{-2}$ \\
4 & $1.9-3.0$ & 2.0 & $(4.72 \pm 0.84) \times 10^{-3}$ \\
5 & $3.0-4.5$ & 2.0 & $(3.05 \pm 0.80) \times 10^{-3}$ \\
\hline
\end{tabular}

to the wide PSF wings these sources generate a residual signal also above $r=30^{\prime \prime}$. This has been removed in the same way as described by Sołtan (2006). Consequently, the amplitude variations of the $w(r)$ functions shown in Fig. 1 reflect the actual large scale fluctuations of the XRB.

Although the systematic trends of the $w(r)$ function are obvious in all the energy bands, a large scatter of points does not allow for the detailed studies of the $w(r)$ shape. To quantify a slope of the $w(r)$ in all the energy bands, we calculate the average values of $w(r)$ in the ranges of $1^{\prime}-2^{\prime}$ and $4^{\prime}-7.5^{\prime}$. The differences between the $w$ amplitude at these separation bins are given in Table 1. Quoted uncertainties represent only the statistical scatter of points contributing to the average values of $w(r)$. Small amplitude of the $w(r)$ results from the fact that the total count rates include a substantial amount of uniformly distributed noncosmic background (especially in the higher energy bands), and the fluctuations of the XRB are substantially diluted in the raw correlation function (see Eq. (10)).

\section{Sources of the XRB variations}

The total count rates recorded in a single pointing are a mixture of the cosmic signal and various components of the local origin including $\mathrm{X}$-rays produced in the satellite and the particle background. For each observation a contribution of the contaminating counts is different and essentially unknown. In the present investigation the cosmic signal in the individual pointing is not isolated from the total counts. The average contribution of the non-cosmic count rates in the whole set of observations is estimated by comparison of the total count rates in the whole sample with estimates of the cosmic XRB taken from the literature.

Apart from the small scale fluctuations investigated in the present paper, the XRB above $1 \mathrm{keV}$ is highly isotropic and well approximated by a power law. Both the normalization and slope have been determined in the past by several groups using different instruments. In the present analysis we use parametrization of the XRB by Lumb et al. (2002) with the photon spectral index $\Gamma=-1.42$ and the normalization at $1 \mathrm{keV}$ of $9.0 \mathrm{ph} \mathrm{keV}^{-1} \mathrm{~cm}^{-2} \mathrm{~s}^{-1} \mathrm{sr}^{-1}$. Below $1 \mathrm{keV}$ some fraction of the $\mathrm{XRB}$ is produced by the hot gas in the Galaxy and the WHIM. Galactic contribution to the sub-keV background exhibits variations over the celestial sphere, but one should expect that in the arcmin scales this component is constant. The pointings used in our investigation are scattered over a large area at high latitudes and it is justified to use the average XRB spectrum derived by Lumb et al. (2002). In the energy ranges of two soft bands $0.3-0.5 \mathrm{keV}$ and $0.7-1 \mathrm{keV}$ the cosmic spectrum is adequately approximated by a power law with the photon index $\Gamma=-2.78$.

The broken power law with these spectral indices is used to calculate the expected XRB count rates in the EPIC MOS observations in five energy bands. These count rates are listed in Table 2 together with the average total count rates actually observed in the data. 

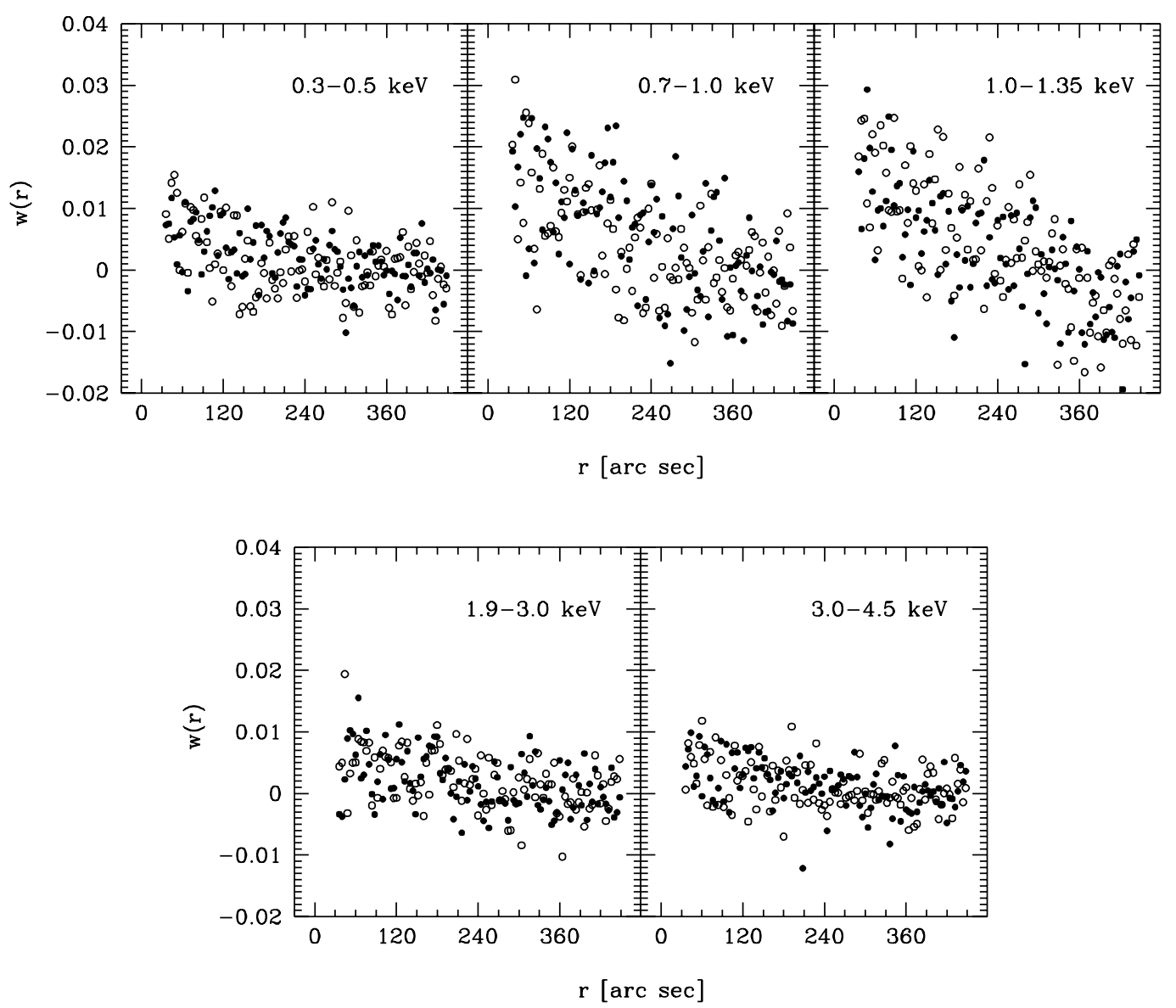

Fig. 1. The raw ACF as defined in Eq. (8). Open symbols - MOS 1, filled - MOS 2.

Table 2. Total observed and model XRB count rates.

\begin{tabular}{llcccccc}
\hline \hline$\#$ & $E[\mathrm{keV}]$ & & \multicolumn{2}{c}{${\text { Count Rates }\left[\mathrm{cnt} \mathrm{s}^{-1} \mathrm{pxl}^{-1}{ }^{a}\right]}$} & $\Delta \xi_{\mathrm{PL}}$ \\
& & Total Observed $^{b}$ & Total Cosmic $^{c}$ & Power Law $^{d}$ & WHIM $^{e}$ & AGN+clusters $^{f}$ & \\
\hline 1 & $0.3-0.5$ & $1.13 \times 10^{-6}$ & $7.9 \times 10^{-7}$ & $2.3 \times 10^{-7}$ & $(5.2 \pm 1.1) \times 10^{-8}$ & $4.8 \times 10^{-7}$ & $0.1419 \pm 0.0228$ \\
2 & $0.7-1.0$ & $7.27 \times 10^{-7}$ & $4.7 \times 10^{-7}$ & $3.7 \times 10^{-7}$ & $(4.1 \pm 0.8) \times 10^{-8}$ & $4.3 \times 10^{-7}$ & $0.0445 \pm 0.0058$ \\
3 & $1.0-1.35$ & $6.73 \times 10^{-7}$ & $4.2 \times 10^{-7}$ & $4.2 \times 10^{-7}$ & $(4.8 \pm 7.1) \times 10^{-9}$ & $4.2 \times 10^{-7}$ & $0.0337 \pm 0.0035$ \\
4 & $1.9-3.0$ & $9.81 \times 10^{-7}$ & $3.8 \times 10^{-7}$ & $3.8 \times 10^{-7}$ & 0 & $3.8 \times 10^{-7}$ & $0.0310 \pm 0.0055$ \\
5 & $3.0-4.5$ & $1.04 \times 10^{-6}$ & $2.9 \times 10^{-7}$ & $2.9 \times 10^{-7}$ & 0 & $2.9 \times 10^{-7}$ & $0.0383 \pm 0.0101$ \\
\hline
\end{tabular}

${ }^{a} \mathrm{pxl}=4^{\prime \prime} \times 4^{\prime \prime} ;{ }^{b}$ count rates averaged over all the pointings including non-cosmic background; ${ }^{c}$ best estimate approximated by the broken power law (see text); ${ }^{d}$ with photon index $\Gamma=-1.42$ and absorption by the cold gas with $N_{\mathrm{H}}=2.2 \times 10^{20} \mathrm{~cm}^{-2} ;{ }^{e}$ count rates actually detected (lower limit for the total WHIM emission); ${ }^{f}$ extragalactic component excluding WHIM.

\subsection{Fluctuations above $1 \mathrm{keV}$}

The XRB above $\sim 1 \mathrm{keV}$ is generated virtually exclusively by the extragalactic discrete sources. Most of the flux comes from a whole variety of AGNs. A poorly constrained fraction of the $\mathrm{XRB}$ is produced by clusters of galaxies. Neither theoretical considerations, nor observations indicate that a measurable contribution results from the WHIM emission at these energies. It is also established that plasma in the Galaxy does not contribute substantially to XRB above $1 \mathrm{keV}$ (e.g. Galeazzi et al. 2007; Henley et al. 2007). A discrete nature of the XRB at these energies has implications for the XRB structure. Assuming that the specific population of objects generates a constant fraction of the XRB in the consecutive energy bands, the relative amplitude of the XRB fluctuations should also be constant. In the following we use this conjecture to analyze the AGN contribution to the XRB.
Count rates generated by the power law spectrum with $\Gamma=$ -1.42 and the Lumb et al. (2002) normalization are listed in Table 2. The low energy absorption by a cold gas in the Galaxy has been accounted for assuming the average in the sample hydrogen column density of $2.2 \times 10^{20} \mathrm{~cm}^{-2}$. In all five energy bands the power law count rates are substantially lower than the total average count rates recorded in the sample. In three high energy bands these differences result solely from the non-cosmic counts, while in two low energy bands the effect of the plasma emission is also visible. Substituting into Eq. (10) the "total observed" and "power law" count rates given in the Table 2 and $\Delta w$ from Table 1 we get the expected increments of the ACF, $\Delta \xi_{\mathrm{PL}}$, under the assumption that all the XRB fluctuations are produced solely by the discrete extragalactic sources generating the power law component. The model amplitudes of $\Delta \xi_{\mathrm{PL}}$ are listed in the last column in Table 2. For three energy bands 


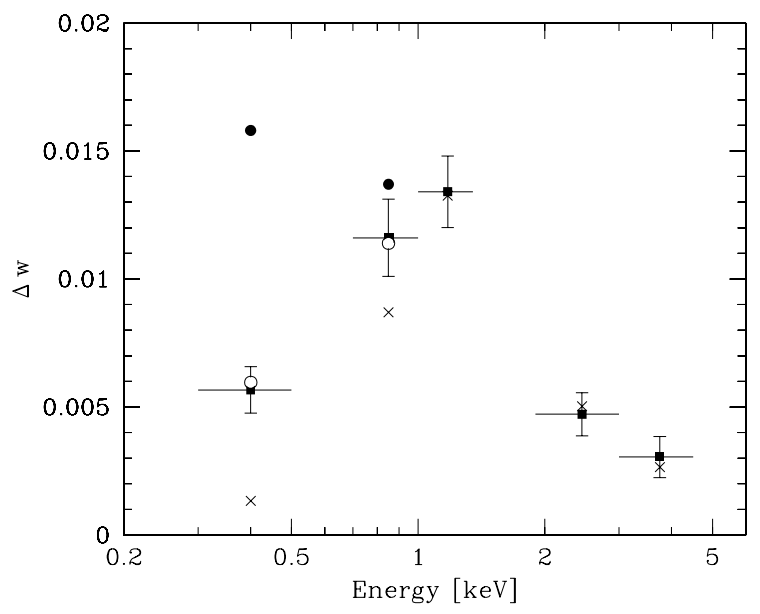

Fig. 2. The raw ACF increment $\Delta w$ - points with error bars. Models of $\Delta w$ distribution assuming $\Delta \xi=0.033$ : crosses - for fluctuations of power law component of the XRB; dots - for fluctuations of the whole XRB; open circles - for fluctuations of the XRB component described by the broken power law (see text for details).

above $1 \mathrm{keV}$, the ACF signal is consistent with the constant value of $\Delta \xi_{\mathrm{PL}}=0.033$. However, the model at lower energies requires much larger amplitudes of $\Delta \xi_{\mathrm{PL}}$. It implies that the simple power law model is unable to explain the total observed amplitude of the XRB fluctuations over the entire energy range.

The relationship between the fluctuations of the raw count rates, $\Delta w$, and the power law model is visualized in Fig. 2. Here the model ACF amplitude, $\Delta \xi$, is used to reproduce the observed fluctuations, $\Delta w$. In agreement with the above conclusion, the ACF amplitude $\Delta \xi_{\mathrm{PL}}=0.033$ fits perfectly the $\Delta w$ values in three high energy bands and fails to reproduce the observed $\Delta w$ signal in two low energy bands. Higher fluctuation amplitude below $1 \mathrm{keV}$ indicates that either the extragalactic component of the XRB is not adequately represented by a single power law, or a substantial fraction of fluctuations is generated by the WHIM.

\subsection{WHIM contribution to the soft XRB}

Observational constraints on the WHIM contribution to the soft $\mathrm{XRB}$ are rather weak. The lower limit for the total WHIM emission is defined by the amplitude of the WHIM signal correlated with the sample of galaxies as measured by Sołtan (2006). In his paper, the extended emission in all five energy bands correlated with galaxies in the local universe was determined. The galaxies used in the investigation constituted a sample of well-defined statistical characteristics with a surface density of 340 objects per sq. deg. The correlated signal corrected for the residual cluster contribution (see the original paper for details) was used to estimate the mean emission per galaxy in the sample. This mean flux is used here to calculate the average WHIM surface brightness which is given in Table 2. Quoted errors represent only statistical uncertainties. The WHIM signal in two low energy bands is detected at better than $4 \sigma$ level and constitutes nearly $7 \%$ of the XRB in the band $0.3-0.5 \mathrm{keV}$ and $9 \%$ in $0.7-1.0 \mathrm{keV}$. These figures represent only the lower limit for the WHIM emission, since basically unknown additional flux could be potentially generated by the WHIM surrounding galaxies not represented in the original sample.

The absolute upper limit for the WHIM flux is obtained by minimizing the contributions due to the galactic plasma and discrete extragalactic sources. Assuming that below $1 \mathrm{keV}$ the average AGN spectrum could still be approximated by the power law with $\Gamma=-1.42$ and absorption by a cold gas, the maximum count rate of the WHIM in the $0.7-1.0 \mathrm{keV}$ band amounts to $\sim 1 \times 10^{-7} \mathrm{cnt} \mathrm{s}^{-1} \mathrm{pxl}^{-1}$, or $21 \%$ of the XRB (pxl = $\left.4^{\prime \prime} \times 4^{\prime \prime}\right)$. This figure is obtained assuming negligibly small galactic emission in the band. Assuming further that the total WHIM emission has a spectral shape similar to the emission correlated with the galaxies investigated by Sołtan (2006), we find that the maximum count rate possibly attributed to the WHIM in the lowest energy band of $0.3-0.5 \mathrm{keV}$ amounts to $\sim 1.3 \times$ $10^{-7} \mathrm{cnt}^{-1} \mathrm{pxl}^{-1}$, or $16 \%$ of the XRB.

Although the WHIM emission correlated with galaxies generates a relatively strong signal in the CCF, the WHIM contribution to the ACF of the integral background is expected to be small. The available data are insufficient to determine precisely the ACF amplitude due to the WHIM. Nevertheless, the WHIM properties as determined by Sołtan (2006) provide some estimates on the X-ray fluctuation amplitude generated by the intergalactic medium. The average X-ray surface brightness enhancement around a randomly chosen galaxy in the sample investigated by Sołtan (2006) does not exceed $\delta \rho=3 \times$ $10^{-8} \mathrm{cnt} \mathrm{s}^{-1} \mathrm{pxl}^{-1}$ in the two low energy bands and is lower in the remaining bands. So, it is below $3 \%$ of the raw count rate in the $0.3-0.5 \mathrm{keV}$ and is around $4 \%$ in the $0.7-1.0 \mathrm{keV}$ band. Since the amplitude of the ACF $w \sim(\delta \rho /\langle t\rangle)^{2}$, the WHIM contribution to the increment of $w$ could be below 0.001 and 0.002 in these two bands, respectively. These figures are smaller than uncertainties of our $\Delta w$ estimates shown in Fig. 2 and could be neglected in the present considerations. One should note, however, that allowing a moderate scatter of the halo luminosities, the ACF signal generated by the WHIM would exceed the above estimates. Although the low contribution of the WHIM to the fluctuations is favored, the potential effect of the WHIM ACF uncertainties is accounted for in the following calculations.

\subsection{Fluctuations below $1 \mathrm{keV}$}

A simple model of the XRB fluctuations generated by a population of sources with a power law spectrum provides an amplitude which is too low for the fluctuations in the sub-keV domain. The discrepancy between the observed amplitude $\Delta w$ and the model implies that either the actual contribution of AGNs to the XRB below $1 \mathrm{keV}$ is higher than the pure power law, or the amplitude of the WHIM fluctuations has been underestimated.

One should note, however, that the present investigation confirms a well established fact that the soft XRB cannot be produced entirely by AGNs, but some fraction of the XRB is generated by a smoothly distributed gas in the Galaxy. The total cosmic count rates and $\Delta \xi=0.033$ would generate the fluctuations of the raw counts substantially above the observed amplitude in two low energy bands. This is shown in Fig. 2 with full dots. Clearly, only a fraction of sub-keV counts is distributed according to $\Delta \xi=0.033$, while the remaining counts are distributed more uniformly.

Below we consider two extreme cases of the WHIM contribution to the XRB fluctuations. If the WHIM emission generates weak fluctuations of the raw counts, only the discrete extragalactic sources could generate the observed $\Delta w$ amplitude. In this case, the requirement that the observed fluctuations below $1 \mathrm{keV}$ are reproduced by $\Delta \xi=0.033$ implies a softer source spectrum than that at higher energies. Assuming the power law we get the photon index of -2.25 . The model which fits the data is shown in Fig. 2 with open circles and the corresponding count rates are given in the Table 2. A conclusion that the fluctuating XRB 
component exhibits soft excess is in agreement with the steepening of the AGN spectra below $1 \mathrm{keV}$ observed in individual Xray bright objects (see for example Elvis et al. 1994). A similar effect is also visible in the population of weaker sources which generate $\sim 30 \%$ of the XRB at $2 \mathrm{keV}$ (Mateos et al. 2005). It is possible, however, that this coincidence is fortuitous, since the $\mathrm{XRB}$ spectrum is significantly harder than the average spectrum of the AGNs investigated by these authors. Thus, the remaining $70 \%$ of the XRB is produced by still weaker sources which do not necessarily exhibit the soft excess.

If the integrated AGN contribution to the XRB below $1 \mathrm{keV}$ does not exceed the power law extrapolated from the higher energies, the fluctuation amplitude in the sub-keV domain could be generated by poor clusters or groups of galaxies with masses below $\sim 2 \times 10^{13} M_{\odot}$. Since the gas temperature in these objects is typically lower than $1 \mathrm{keV}$ (Horner et al. 1999), their extended emission contributes to the fluctuations at scales of a few arcmin merely in the soft energy bands. Unfortunately, the present estimates of the ACF shape are inadequate to distinguish between different fluctuation mechanisms and we are unable isolate the cluster contribution.

The soft excess observed in the extragalactic component reduces estimates of the galactic signal. The difference between the total cosmic signal and the extragalactic component provides an estimate of the mean galactic contribution to the XRB. The extragalactic broken power law together with the observed WHIM flux generate all the XRB in the $0.7-1.0 \mathrm{keV}$ band and $67 \%$ of the XRB in the $0.3-0.5 \mathrm{keV}$ band. Thus, the galactic component visible only in the softest band, is substantially softer than what is usually assumed.

The relationship between various components is not significantly changed if the average spectrum of extragalactic discrete sources over the entire energy range is power law with $\Gamma=-1.42$ and the "missing" $\Delta w$ amplitude below $1 \mathrm{keV}$ is generated by the WHIM. In this case the galactic plasma would contribute up to $65 \%$ in the $0.3-0.5 \mathrm{keV}$ band and to $13 \%$ in the $0.7-1.0 \mathrm{keV}$ band. Although this contribution is substantially higher than in the broken power law case, the galactic spectrum is still very soft.

\section{Discussion and conclusions}

Absolute fluxes of the major components of the soft XRB are investigated using the information extracted from the correlation functions. The variations of the correlation amplitude with photon energy have allowed us to separate the fluctuating extragalactic component from the smooth (although also variable at large scales) galactic emission. The total fluctuations above $1 \mathrm{keV}$ are adequately described by a single ACF. This conclusion is in agreement with a well-established fact that at high energies the XRB is generated entirely by the extragalactic discrete sources (AGNs and clusters). One should note that the present estimate of the increment, $\Delta \xi=0.033$, is in good agreement with the Sołtan et al. (2001) measurement of the XRB fluctuations based on the ROSAT pointings (Sołtan 2007).

In calculations below $1 \mathrm{keV}$ we apply the fluctuation analysis without any a priori assumption on the spectral shape of both components, while in the "canonical" approach to the question of the galactic contribution, the power law spectrum of the extragalactic component is usually assumed (cf. Galeazzi et al. 2007; Henley et al. 2007). This is justified at energies above $1 \mathrm{keV}$, but in the sub-keV region it is likely that the power law approximation fails to represent the average spectrum of AGNs.
The WHIM contribution to the total XRB flux is distinctly smaller than the AGN component. But the soft WHIM spectrum substantially affects the XRB budget below $1 \mathrm{keV}$. Because the galactic emission is not easily distinguished from the WHIM signal, the soft section of the XRB was frequently attributed to the galactic plasma ignoring the genuine contribution by the WHIM. The lower limit for the WHIM flux is determined by the crosscorrelation of the XRB surface brightness with the local galaxy distribution (Sołtan 2006). The total WHIM contribution to the $\mathrm{XRB}$ is assessed at $7-16 \%$ in $0.3-0.5 \mathrm{keV}$ band and $9-21 \%$ in $0.7-1.0 \mathrm{keV}$. In the sub-keV region both galactic hot plasma and the WHIM contribute to the XRB. Nevertheless, in the band of $0.7-1.0 \mathrm{keV}$, the AGNs generate still at least $79 \%$ of the total XRB flux. The extragalactic discrete source contribution drops significantly below $0.5 \mathrm{keV}$, but even in the softest band it is likely that AGNs make up to $60 \%$ of the background.

It is estimated that the galactic emission averaged over many pointings scattered in two galactic hemispheres generates $33 \%$ of the total cosmic signal in the softest band (although a higher value is not ruled out in the present investigation). The galactic contribution drops sharply with the energy. In the $0.7-1.0 \mathrm{keV}$ band it amounts to at most $13 \%$ of the XRB, and the data are consistent with no detectable galactic signal. It appears that the mean temperature of the emitting plasma does not significantly exceed $10^{6} \mathrm{~K}$. This roughly corresponds to the estimates of the temperature in the Local Bubble, but is inconsistent with the hot galactic halo model. According to the standard approach, the entire excess of the soft XRB above the power law extrapolation is attributed to the Galaxy. Detailed studies by several groups have measured the Local Bubble emission and estimated its temperature at about $10^{6} \mathrm{~K}$. Thermal emission of such plasma, however, cannot explain the signal between $\sim 0.6$ and $1 \mathrm{keV}$. To account for the excess flux in this energy range, it is postulated that the Galaxy is surrounded by the halo of hot plasma with the temperatures close to $3 \times 10^{6} \mathrm{~K}$. Our analysis indicates that the amplitude of the hot halo emission is substantially smaller than is usually assumed. The discrepancy between the present conclusion and earlier estimates, results directly from the analysis of the WHIM emission and the different estimates of the extragalactic component.

Acknowledgements. I thank all the people involved in the XMM-Newton project for making the XMM Science Archive and Standard Analysis System such userfriendly environment. This work has been partially supported by the Polish KBN grant 1 P03D 00327.

\section{References}

Cen, R., \& Ostriker, J. P. 1999, ApJ, 514, 1

Bryan, G. L., \& Voigt, G. M. 2001, ApJ, 556, 590

Croft, R. A. C., Di Matteo, T., Davé, R., et al. 2001, ApJ, 557, 67

Davé, R., Cen, R., Ostriker, J. P., et al. 2001, ApJ, 552, 473

Elvis, M., Wilkes, B. J., McDDowell, J. C., et al. 1994, ApJS, 95, 1 Galeazzi, M., Gupta, A., Covey, K., \& Ursino, E. 2007, ApJ, 658, 1081 Henley, D. B., Shelton, R. L., \& Kuntz, K. D. 2007, ApJ, 661, 304 Horner, D. J., Mushotzky, R. F., \& Scharf, C. A. 1999, ApJ, 520, 78 Kim, M., Wilkes, B. J., Kim, D.-W., et al. 2007, ApJ, 659, 29 Lehmann, I., Hasinger, G., Schmidt, M., et al. 2001, A\&A, 371, 833 Lumb, D. H., Warwick, R. S., Page, M., \& De Luca, A. 2002, A\&A, 389, 93 McCammon, D., Almy, R., Apodaca, E., et al. 2002, ApJ, 576, 188 Mateos, S., Barcons, X., Carrera, F. J., et al. 2005, A\&A, 444, 79 Nevalainen, J., Markevitch, M., \& Lumb, D. 2005, ApJ, 629, 172 Sołtan, A. M. 2006, A\&A, 460, 59

Sołtan, A. M. 2007, in Pathways through an eclectic Universe, ed. J. H. Knapen,

T. J. Mahoney, \& A. Vazdekis, ASP Conf. Ser., in preparation

Sołtan, A. M., Freyberg, M., \& Trümper, J. 2001, A\&A, 378, 735

Sołtan, A. M., Freyberg, M., \& Hasinger, G. 2002, A\&A, 395, 475

Sołtan, A. M., Freyberg, M., \& Hasinger, G. 2005, A\&A, 436, 67 